\title{
Erratum to: Induction on differentiation and modulation of bone marrow progenitor of dendritic cell by methionine enkephalin (MENK)
}

Jinling Liu • Wenna Chen • Jingjuan Meng •

Changlong Lu $\cdot$ Enhua Wang $\cdot$ Fengping Shan

Published online: 25 September 2012

(c) Springer-Verlag Berlin Heidelberg 2012

Erratum to: Cancer Immunol Immunother

DOI 10.1007/s00262-012-1221-9

The corresponding author would like to correct the misspelled first author name in the published article. The correct name should be Jinling Liu.

The online version of the original article can be found under doi:10.1007/s00262-012-1221-9.

J. Liu · W. Chen · J. Meng · C. Lu · F. Shan $(\bowtie)$

Department of Immunology, School of Basic Medical Science,

China Medical University, No. 92, North Second Road,

Heping District, Shenyang 110001,

People's Republic of China

e-mail: fpshan@mail.cmu.edu.cn

J. Liu

College of Animal Science and Veterinary Medicine,

Shenyang Agricultural University,

No. 120, Dongling Road, Shenhe District,

Shenyang 110866, People's Republic of China

E. Wang

Institute of Pathology and Pathophysiology,

School of Basic Medical Science, China Medical University,

No. 92 ,

North Second Road, Heping District,

Shenyang 110001, People's Republic of China 